\title{
Impacto das Configurações Familiares no Desenvolvimento de Crianças e Adolescentes: Uma Revisão da Produção Científica
}

\author{
Débora de Oliveira \\ Aline Cardoso Siqueira \\ Débora Dalbosco Dell'Aglio \\ Rita de Cássia Sobreira Lopes \\ Universidade Federal do Rio Grande do Sul
}

\begin{abstract}
RESUMO
O presente estudo teve por objetivo mapear a produção científica sobre o impacto das diferentes configurações familiares, no desenvolvimento de crianças e adolescentes. Foram selecionados abstracts de periódicos científicos nacionais e internacionais indexados em diferentes bases de dados, através de combinações de descritores. A revisão dos abstracts resultou em 415 artigos produzidos nos períodos de 1955 a 2005, sendo que 401 internacionais e 14 nacionais. Observou-se um maior número de publicações a partir da década de 1990. Na literatura internacional, houve predomínio de estudos empíricos, de caráter quantitativo. Estes focalizavam, em sua maioria, famílias monoparentais e adolescentes, em comparação com crianças. Os resultados também revelaram uma maior freqüência de estudos que não encontraram mudanças e conseqüências significativas de diferentes configurações familiares para o desenvolvimento de crianças e adolescentes. No entanto, percebeu-se que, entre os estudos que identificaram mudanças e conseqüências significativas, houve um predomínio das negativas em relação às positivas, o que faz pensar na estigmatização de configurações diferentes da família nuclear. Ainda que a análise tenha se baseado apenas em abstracts, acredita-se que permitiu vislumbrar o estado da arte sobre o tema.
\end{abstract}

Palavras-chave: diferentes configurações familiares; crianças, adolescentes; "estado da arte".

\section{ABSTRACT \\ The Impact of Family Configurations on Child and Adolescent Development: The Review the Scientific Production}

The present study aimed to review the scientific production on the impact of different family configurations on child and adolescent development. Abstracts from national and international periodicals, indexed in different databases, through combinations of keywords, were selected. The review identified 415 articles printed between 1955 and 2005, 401 of which were international and 14 were national. There was a greater number of publications from the 1990's. In the international literature, there was a predominance of quantitative, empirical studies. Most of them focused on single-parent families, and on adolescents as opposed to children. The results also revealed a greater frequency of studies which did not find significant changes and consequences of the different family configurations for child and adolescent development. However, in studies which did identify significant changes and consequences, focus on the negative was more frequent than the positive, which raises the question of stigmatization of family configurations that are different from the nuclear family. Even though the analysis was based on abstracts alone, it is believed that it allowed for the characterization of the state of art in the area.

Keywords: different family configurations; children; adolescents; "state of art".

A família constitui-se em um centro formador da sociedade, bem como do desenvolvimento individual e da maturidade emocional de cada indivíduo (Winnicott, 1965/2001). Família pode ser entendida como um conjunto de relações caracterizadas por influência recíproca, direta, intensa e duradoura entre seus mem- bros (De Antoni, 2005). É o principal agente de socialização da criança, que influencia na aquisição de suas habilidades, comportamentos e valores apropriados para cada cultura, constituindo-se em uma dimensão essencial na vida dos indivíduos. A família caracterizase como parte essencial na construção da saúde emo- 
cional de seus membros, tendo como função básica a proteção e o bem-estar destes (Minuchin, 1982; Osório, 1992).

A instituição família vem sofrendo modificações através da história, que pode variar de uma cultura para outra. Apesar das mudanças, a família apresenta capacidade de sobrevivência e de adaptação, originando diferentes formas de composições e de padrões relacionais (Wagner, 2002). Para Figueira (1986), houve um processo de modernização da família guiado pelo ideal de uma família "igualitária" em detrimento de um ideal hierárquico, herança do sistema patriarcalista. As transformações paradigmáticas, ocorridas principalmente a partir de meados do século $\mathrm{XX}$, no que diz respeito à configuração e ao funcionamento familiar, provocaram alterações na estrutura e na dinâmica de suas relações, contribuindo para a concepção contemporânea de família. Essas modificações têm acarretado mudanças nos padrões de funcionamento entre os seus membros, levando a um processo de assimilação e de construção de novos modos de relacionamento (Wagner, Ribeiro, Arteche \& Bornholdt, 1999).

Em decorrência disso, a família da atualidade é caracterizada por redefinições de papéis, hierarquia e sociabilidade, permitindo diferentes configurações familiares, que estão centradas na valorização da solidariedade, da fraternidade, na ajuda mútua, nos laços de afeto e de amor (Fonseca, 2002; Perrot, 1993; Rizzini, 2002), em detrimento da exigência da consangüinidade (Fachin, 2001; Goldani, 1994). Segundo Osório (2002), diferentes configurações consistem no modo como se dispõem e se inter-relacionam os membros de uma mesma família, que estabelecem relações não apenas por laços consangüíneos. Assim, observa-se que o entendimento atual de família não está mais atrelado necessariamente à concepção de família nuclear, composta de mãe, pai e filhos, seguindo o modelo tradicional patriarcal. A noção de família tem se ampliado na medida em que procura incluir e compreender as diferentes relações entre os seus membros. No cotidiano, é possível encontrar famílias seguindo modelos tradicionais, casais dividindo os cuidados dos filhos e da organização familiar, mulheres e homens assumindo sozinhos o sustento financeiro da família/ produções independentes (monoparentalidade), famílias reconstituídas/recasadas, casais sem filhos, casais homossexuais, casal com filhos adotivos, entre outras.

Embora essas modificações estruturais na organização familiar tenham aumentado o interesse pelo estudo da família nas últimas décadas, com o intuito de compreendê-las e de avaliar os seus efeitos sobre os membros envolvidos (Ramires, 2004; Wagner, 2002), ainda não são conclusivas as suas conseqüências no desenvolvimento de crianças e adolescentes (Amato, 1995). O IBGE aponta que, especificamente, o aumento de separações e divórcios (de 1991 a 2002, houve um crescimento de 59,6\%) e de recasamentos (de 1991 a 2002, houve aumento de 5,5\%) têm feito com que muitas crianças e adolescentes não cresçam mais na presença de pais biológicos, e convivam com o recasamento destes e com novos irmãos (IBGE, 2002/2003). Algumas vezes, essas famílias enfrentam problemas associados às desvantagens econômicas, exigindo de pelo menos um dos progenitores uma maior energia e tempo, não só no trabalho, mas também nas atividades domésticas e de cuidado com os filhos (Dessen \& Braz, 2005). Estes aspectos acabam interferindo na qualidade das relações e no tempo que os pais permanecem com os filhos, exigindo modificações na rotina diária em decorrência da reorganização familiar. Para Ramires (2004), as alterações na vida afetiva, social e profissional dos pais exigem, por vezes, mudanças na rede de convivência e de apoio das crianças, introduzindo a necessidade de relacionamentos com seus familiares e com novos parceiros.

$\mathrm{Na}$ literatura, alguns autores apontam o prejuízo e o sofrimento atrelados à separação e ao divórcio, entendendo-os como um choque doloroso e angustiante (Carter \& McGoldrick, 1989/2001; Wallestein, 1985), já que podem desencadear problemas de ajuste psicológico e social nas crianças e adolescentes (Walsh, 1993). Outros autores destacam o seu potencial de reorganização e o amadurecimento precoce destes, que tendem a expressar mais atitudes amorosas com os pais quando comparados aos filhos de famílias intactas (Parish \& Necessary, 1994). Segundo Hetherington e Stanley-Hagan (1999), crianças de famílias monoparentais podem apresentar maior nível de bem-estar do que as de famílias nucleares com alto nível de conflito, uma vez que o bem-estar infantil está associado ao bem-estar dos pais e ao relacionamento entre eles.

Além do nível de conflito do ambiente familiar, o desenvolvimento cognitivo e das estruturas de pensamento das crianças também podem influenciar na compreensão e nas respostas frente ao divórcio dos pais (Ramires, 2002). Crianças de oito a nove anos de idade tendem a apresentar maior vulnerabilidade às transições familiares, indicando maiores dificuldades e estresse para lidar com esse momento do divórcio em função do grau de maturidade de seu desenvolvimento 
cognitivo e socioemocional (Kaslow \& Schwartz, 1987/ 1995; Ramires, 2004; Wallerstein \& Kely, 1996/ 1998).

Outros pesquisadores também apontam que as dificuldades de funcionamento familiar não estão, necessariamente, associadas à sua configuração, mas sim às relações que se estabelecem entre os seus membros (Grossman \& Rowat, 1995). A competência ou saúde da família depende de fatores como o desempenho de papéis específicos e a delimitação da função de autoridade nas figuras parentais, a qual é fundamental para um funcionamento familiar saudável e para o bemestar de seus membros (Féres-Carneiro, 1992; FéresCarneiro, 1998). Além disso, a qualidade da comunicação estabelecida entre os cônjuges e a satisfação conjugal também constituem preditores de uma boa saúde física e emocional das crianças (Erel \& Burman, 1995; Féres-Carneiro, 2003; Kreppner, 2000).

Em função da pluralidade de relações afetivas e de configurações familiares e de seu impacto no desenvolvimento de crianças e adolescentes, faz-se necessário conhecer e sistematizar as pesquisas nesse campo de conhecimento. Dessa forma, o objetivo do presente estudo foi mapear a produção científica sobre as famílias separadas/divorciadas, recasadas e monoparentais a partir de estudos nacionais e internacionais de periódicos científicos indexados, apontando a tendência da produção científica, as escolhas metodológicas e as características das amostras desses estudos. Além disso, buscou-se discutir os principais aspectos que vêm sendo investigados e destacados em diferentes décadas.

\section{MÉTODO}

A opção metodológica de pesquisa de levantamento e de avaliação do conhecimento sobre determinado tema é um desafio para o pesquisador que busca conhecer o já construído e produzido para, somente depois, dedicar-se a temas ainda não explorados no campo científico (Ferreira, 2002). Segundo esta auto$\mathrm{ra}$, as pesquisas de caráter bibliográfico são conhecidas pela denominação de "estado da arte" ou "estado do conhecimento". Nos últimos 15 anos, há um destaque significativo de pesquisas que realizam uma descrição da produção acadêmica e científica sobre determinado tema. Dentro dessa perspectiva, o levantamento e a avaliação de abstracts constituem um meio de vislumbrar com maior abrangência a literatura científica, promovendo a divulgação e a facilitação do acesso aos dados estudados.

Esta pesquisa incluiu a produção científica dos últimos 50 anos, das seguintes bases de dados: Medline,
Lilacs, Psyc-Info, Scielo e Bvs-Psi. Os descritores utilizados para o levantamento foram: família, configuração familiar, divórcio, recasamento, separação, monoparentalidade, família monoparental, família reconstituída, crianças e adolescentes, em português; e em inglês, family, family configuration, single-parent family, children, single-family, adolescents, separation, divorce, remarriage, remarried, blended family e stepfamily. Buscou-se utilizar diferentes combinações dessas palavras, com o objetivo de realizar um levantamento abrangente que incluísse o maior número de estudos da área. Dessa forma, somente os estudos que focalizaram os temas da separação/divórcio, recasamento, monoparentalidade e seus efeitos sobre o desenvolvimento de crianças e adolescentes foram selecionados nesse levantamento. Foram identificados 415 abstracts de artigos científicos indexados, entre o período de 1955 a 2005, sendo 401 internacionais e 14 nacionais. A identificação das informações e o levantamento de categorias de análise dos dados foram realizados em duas etapas. As categorias de análise foram elencadas previamente, buscando identificar quantitativamente três informações: o ano de publicação, as características da amostra e o método utilizado. Após esta etapa, os abstracts encontrados foram analisados qualitativamente, de forma a conhecer os principais temas pesquisados e compreender as tendências observadas da produção. Para os abstracts que não apresentaram as informações investigadas, a categoria de análise foi considerada como não especificada.

\section{RESULTADOS E DISCUSSÃO}

Os resultados foram organizados a partir de quatro categorias de análise dos dados a priori: (1) Publicação de artigos científicos por década; (2) Método e amostra dos artigos publicados; (3) Tipo de configuração familiar abordado nas publicações; (4) Conteúdo temático das publicações. Os abstracts foram classificados separadamente por dois juízes e, em caso de discordância, utilizou-se um terceiro juiz. A seguir, serão apresentadas e discutidas as categorias de análise dos dados.

\section{1) Publicação de artigos científicos por década}

Esta primeira categoria de análise refere-se ao levantamento da produção científica internacional e nacional por década. Observou-se que os estudos sobre os temas em foco surgiram timidamente nas décadas de 1950 e de 1970, havendo um acréscimo progressivo nas décadas de 1980, 1990 e 2000, conforme a Figura 1. 


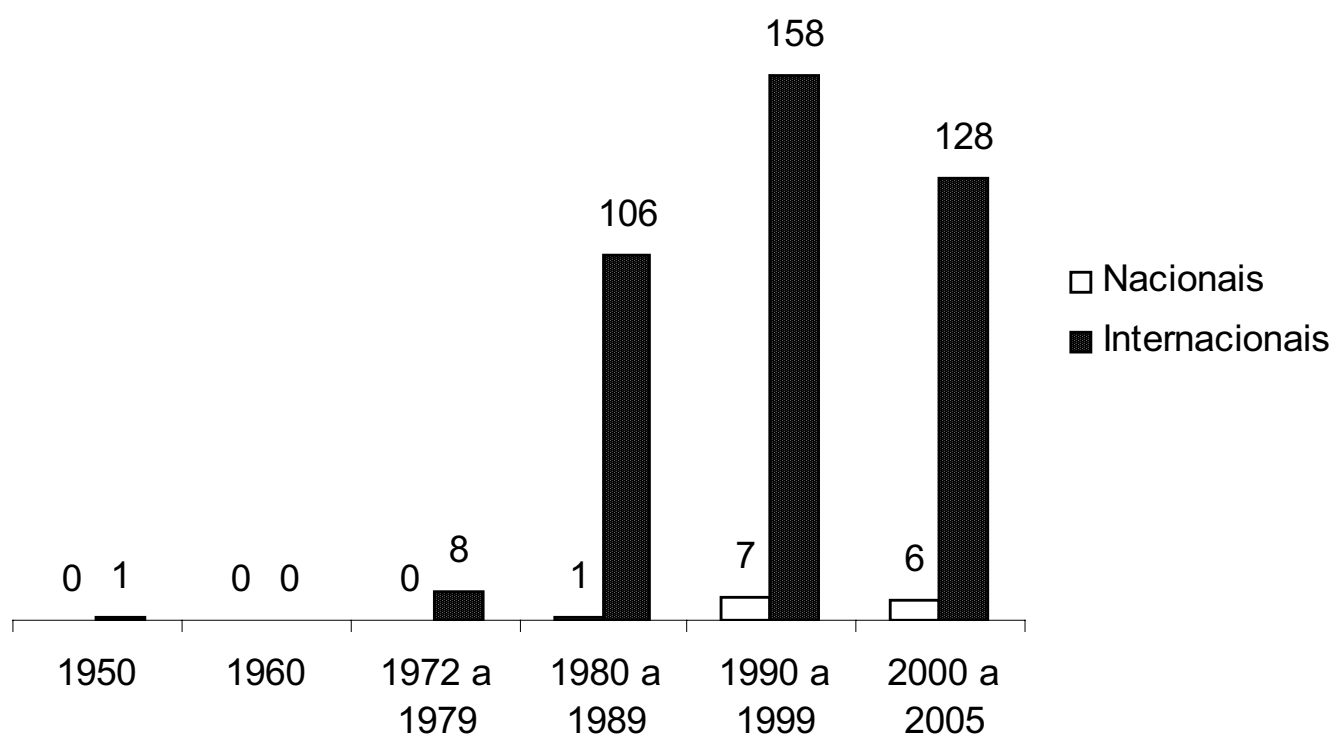

Figura 1. Publicação de artigos internacionais e nacionais por década.

A partir da década de 1980 houve um crescimento abrupto de estudos, no âmbito internacional, sobre famílias separadas/divorciadas, recasadas e monoparentais e os efeitos no desenvolvimento de crianças e adolescentes inseridos nesses contextos. No Brasil, este crescimento foi visualizado na década de 1990. Apesar de pouco expressivo, pode-se constatar que o número de publicações, em um período de cinco anos na década de 2000, é promissor quando comparado à produção de toda década de 1990.

De acordo com Wagner (2002), as pesquisas produzidas, no Brasil, surgiram timidamente na década de 1980, em decorrência da legitimização do divórcio ocorrida em dezembro de 1977 , e enfocaram os chamados "filhos do divórcio". Bucher e Rodrigues (1990) apontam que os temas recasamento e famílias reconstituídas foram pouco investigados na década de 1980 no Brasil, em função do tabu e do obscurantismo que cercavam as relações não legais entre homens e mulheres. Para estes autores, a legitimação da união estável entre homem e mulher como entidade familiar, na nova Constituição Federal de 1988, pode ser considerada um marco importante no incentivo de pesquisas sobre as diferentes configurações familiares. Os valores conservadores e as crenças familiares tradicionais prescritos pela sociedade influenciam na vida dos indivíduos, na medida em que não vivenciar esse padrão de família pode levar ao sofrimento psíquico (Narvaz \& Koller, 2005).

É possível dizer que os resultados observados estão associados ao fato de que, nessas últimas duas décadas, as transformações ocorridas nas configurações familiares provocaram alterações na estrutura e na dinâmica das relações familiares, tanto a nível internacional quanto, especialmente, a nível nacional. Essas transformações no âmbito privado das relações familiares exigiram também uma mudança no campo científico, que passou a ter maior interesse por estes temas, na medida em que se dedicou a descrever as estruturas de parentesco, a analisar as leis referentes à família, as repercussões sobre os indivíduos, dentre outros temas atrelados ao contexto familiar (Dessen \& Braz, 2005; Ramires, 2004; Wagner, 2002). Ainda que em menor número de publicações, quando comparado ao internacional, pesquisadores brasileiros passaram a interessar-se pela compreensão das diferentes configurações familiares e de seus efeitos sobre os membros envolvidos.

\section{2) Método e amostra dos artigos publicados}

Esta categoria de análise refere-se ao levantamento da produção nacional e internacional em termos de 
método (empírico ou teórico; quantitativo e/ou qualitativo) e características da amostra dos estudos entre o período de 1955 a 2005. De acordo com a Tabela 1, observou-se um destaque para os estudos empíricos, tanto em nível internacional $(78,3 \%)$ quanto nacional $(71,42 \%)$.

TABELA 1

Freqüência de Artigos Internacionais e Nacionais por Método Empírico vs. Revisão

\begin{tabular}{ccccccc}
\hline & \multicolumn{2}{c}{ Internacionais } & \multicolumn{2}{c}{ Nacionais } & \multicolumn{2}{c}{ Total } \\
\hline & $f$ & $\%$ & $f$ & $\%$ & $f$ & $\%$ \\
Empírico & 314 & 78,3 & 10 & 71,42 & 325 & 78,31 \\
Revisão & 80 & 19,50 & 4 & 28,57 & 83 & 20 \\
Não-especificado & 7 & 1,74 & - & - & 7 & 1,68 \\
\hline Total & 401 & 100 & 14 & 100 & 415 & 100 \\
\hline
\end{tabular}

Uma análise do tipo de método (quantitativo e/ou qualitativo) foi realizada, conforme mostra a Figura 2. Os abstracts encontrados indicaram em sua descrição de método os seguintes termos: quantitativo, qualitativo e quantitativo-qualitativo. Observou-se também que alguns não especificaram o método utilizado. Em função de a análise do presente estudo centrar-se em abstracts de artigos científicos, não foi possível identificar o tipo de técnica de análise utilizada pelos mesmos. Assim, entende-se por método quantitativo os estudos que testam hipóteses, comparam constructos específicos a partir de grupos de análise (Creswell, 1994). Através do uso desse método, é possível ter uma maior precisão na medida, uma vez que os dados podem ser mais facilmente sumarizados e comparáveis, podendo ser generalizados para além da amostra estudada. Por sua vez, o método qualitativo não procura enumerar ou medir os eventos estudados, prende- se às nuances de sentidos e aos elos lógicos dos relatos, detendo-se ao estudo de um determinado fenômeno (Creswell, 1994). O uso desse método permite muito mais a compressão e a descrição dos dados do que a generalização dos mesmos (Godoy, 1995a; Godoy, 1995b). Já o método quanti-qualitativo, é entendido pelo continuum interativo entre esses dois métodos. Esse entrelaçamento facilita a avaliação, o planejamento e a conduta da pesquisa, de tal modo que maximiza ambas as metodologias (Benz \& Newman, 1998; Creswell, 1994). Os estudos internacionais utilizaram método predominantemente quantitativo $(59,35 \%)$, seguido pelo método qualitativo $(25,93 \%)$ e apenas $1,74 \%$ estudos utilizaram método quanti-qualitativo. Quanto aos estudos nacionais, constatou-se uma preferência pelo método qualitativo $(71,43 \%)$, seguido pelos métodos quanti-qualitativo $(14,28 \%)$ e quantitativo $(14,28 \%)$.

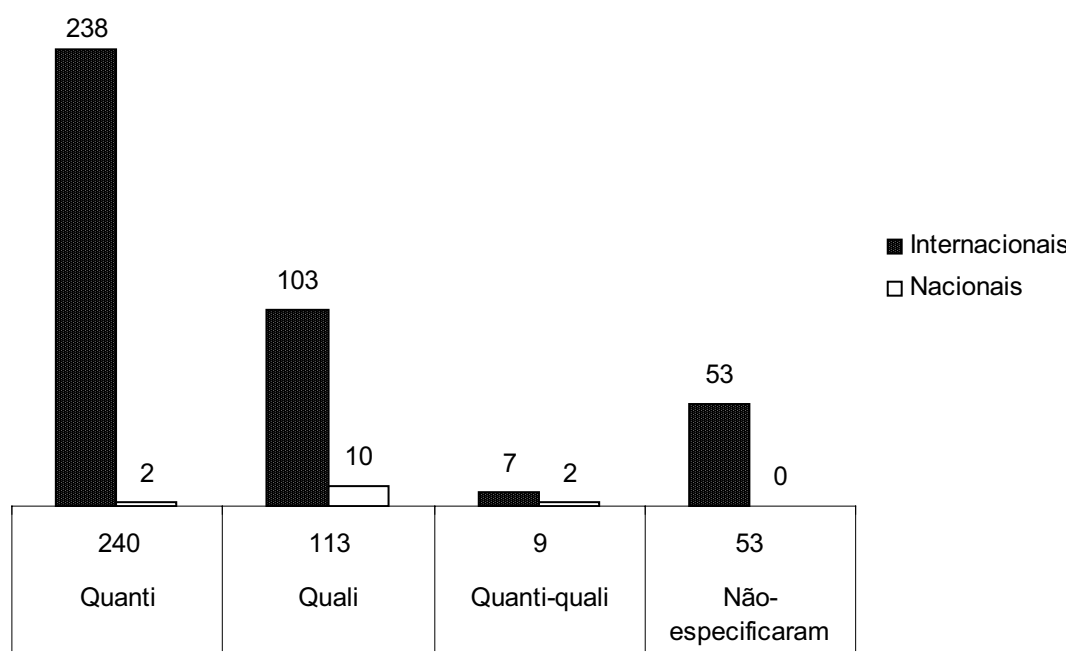

Figura 2. Publicação de artigos internacionais e nacionais por método quantitativo vs. qualitativo. 
No que se refere ao levantamento das características da amostra dos artigos internacionais e nacionais, não foi possível mapear detalhadamente a faixa etária dos participantes, visto que houve muitos estudos que falavam de "filhos", sem especificar a idade, e também outros que incluíram tanto crianças quanto adolescentes. Observou-se uma tendência dos abstracts de não especificarem a faixa etária da amostra de crianças e de adolescentes $(155 ; 100 \%)$. Entre os que especificaram $(260 ; 100 \%)$, observou-se uma tendência de amostras envolvendo adolescentes (109; $41,92 \%)$ e crianças $(95 ; 36,53 \%)$, e de crianças e adolescentes $(56 ; 21,53 \%)$.

De acordo com Bucher e Rodrigues (1990), os estudos enfocando os pais, madrastas, padrastos e a relação conjugal surgiram mais recentemente. É possível dizer ainda que a tendência de focalizar a população de adolescentes, seguidos de crianças também foi observada no contexto brasileiro. A multiplicidade de amostras, indicada pelo levantamento do presente estudo, ressalta a preocupação dos pesquisadores em investigar as diferentes configurações e seus efeitos sobre crianças e adolescentes, a partir de diferentes pontos de vista. O predomínio de estudos com amostras de adolescentes e de crianças pode estar associado ao nível de conflito envolvido nesses momentos de transição familiar, podendo ter efeitos diretos sobre o bem-estar psicológico, desenvolvimento cognitivo e emocional, estruturas de pensamento, relacionamento interpessoal, entre outros aspectos, levando crianças e adolescentes a apresentarem dificuldades para lidar com esta situação (Hetherington \& Stanley-Hagan, 1999; Mazur, 1993; Parish \& Necessary, 1994; Ramires, 2002; Ramires, 2004; Wallestein, 1985; Walsh, 1993; Wagner, 2002; Wagner \& cols., 1999; Wagner, Falcke \& Meza, 1997). Observa-se que estes aspectos são ainda mais focalizados na adolescência, visto que é entendida como uma transição vital, também estando associada a reorganizações sociais, emocionais e cognitivas (Levisky, 1998). Outra hipótese que pode ser pensada para explicar o maior número de pesquisas com adolescentes está relacionada, primeiro, ao estágio de desenvolvimento cognitivo dos adolescentes, que os permite entender os instrumentos e responder escalas auto-aplicativas com maior facilidade, e em segundo, pelo fato dos adolescentes colocarem-se acessíveis e disponíveis para participar de atividades novas, como uma pesquisa.

\section{3) Tipo de configuração familiar abordada nas publicações}

Esta categoria refere-se ao tipo de configuração familiar pesquisado pelos abstracts. A partir dos dados, foram levantados quatro tipos de configuração: (1) monoparentalidade, (2) divórcio/separação, (3) recasamento e (4) diferentes configurações. Entende-se por diferentes configurações os abstracts que tratavam de mais de duas configurações familiares, como família intacta, monoparental e recasada, ou ainda, referiam-se às mesmas sem especificações.

Como pode ser visto na Tabela 2, entre os $a b s$ tracts internacionais, houve um predomínio de estudos sobre monoparentalidade (50,37\%), e entre os abstracts nacionais, o tipo de configuração mais pesquisado foi o divórcio/separação (50\%). Não foi encontrado nenhum estudo sobre monoparentalidade na amostra dos abstracts nacionais. Como o presente estudo mapeou os abstracts indexados em banco de dados, é possível que a produção nacional sobre a monoparentalidade, especificamente, esteja inserida em periódicos não indexados, em capítulos de livros desenvolvidos por grupos de pesquisas em todo o país, ou ainda, em dissertações e teses.

TABELA 2

Freqüência de Artigos Internacionais e Nacionais por Tipo de Configuração Familiar

\begin{tabular}{ccccccc}
\hline & \multicolumn{2}{c}{ Internacionais } & \multicolumn{2}{c}{ Nacionais } & \multicolumn{2}{c}{ Total } \\
\hline & $\mathrm{f}$ & $\%$ & $\mathrm{f}$ & $\%$ & $\mathrm{f}$ & $\%$ \\
Monoparentalidade & 202 & 50,37 & - & - & 202 & 48,67 \\
Divórcio/Separação & 63 & 15,71 & 7 & 50 & 70 & 16,86 \\
Recasamento & 47 & 11,72 & 2 & 14,28 & 49 & 11,8 \\
Diferentes Configurações & 89 & 22,19 & 5 & 35,71 & 94 & 22,65 \\
\hline Total & 401 & 100 & 14 & 100 & 415 & 100 \\
\hline
\end{tabular}

O predomínio da configuração monoparentalidade, no contexto internacional, reflete a preocupação cien- tífica pelo funcionamento dessas famílias chefiadas por apenas uma figura parental e pela forma como 
esta figura está promovendo ou não o desenvolvimento de seus filhos (Grzybowski, 2002). Por outro lado, no contexto nacional, houve maior interesse por famílias recasadas, pelo processo de adaptação familiar a esta nova situação e pelo reflexo no desenvolvimento de crianças e adolescentes. De acordo com Wagner (2002), o recasamento passou a ser considerado uma possibilidade de reconquistar e reconstruir vínculos de intimidade, afeto, companheirismo, promovendo e facilitando a saúde de seus membros. Isto acabou por despertar o interesse de pesquisadores pelo conhecimento do funcionamento desses laços de afeto reconstruídos.

\section{4) Conteúdo temático das publicações}

Esta categoria de análise refere-se aos conteúdos temáticos dos abstracts empíricos e de revisão, focalizando os resultados encontrados, no caso de artigos empíricos, e o tema em discussão, no caso dos artigos de revisão de literatura. Esta análise teve como objetivo avaliar como foram consideradas as diferentes configurações familiares, investigando as mudanças e as conseqüências negativas, positivas, as que não apresentaram mudanças e conseqüências significativas e ainda as que priorizavam uma discussão sobre o tema. Dessa forma, foram identificados quatro eixos, que serão mencionados a seguir.

1) Mudanças e conseqüências negativas: Refere-se aos abstracts que apontaram os prejuízos e as desvantagens ocorridas nas famílias monoparentais, recasadas e divorciadas/separadas no desenvolvimento de crianças e adolescentes. Por exemplo: crianças e adolescentes que vivenciaram divórcio, recasamento e monoparentalidade apresentaram transtornos de conduta (Pollinger, Samuels \& Stadolnik, 2005), hiperatividade (Lalloo, Sheiham \& Nazroo, 2003), transtorno psiquiátrico (Barocas, Seifer \& Sameroff, 1985), problemas de saúde (Weissman, Jogerst \& Dawson, 2003), mais probabilidade de abuso de drogas (Fletcher, 2004) e desvantagens nas competências sociais e acadêmicas (Guidubaldi \& Perry, 1984), entre outros prejuízos.

2) Mudanças e conseqüências positivas: Diz respeito àqueles abstracts que destacaram as oportunidades ocorridas em famílias monoparentais, divorciadas/separadas e recasadas para o desenvolvimento de crianças e adolescentes. Por exemplo, pais de famílias monoparentais utilizaram menos maus-tratos físicos em comparação a famílias com as duas figuras parentais (Kroes, Kalff, Steyaert \& cols., 2002), as crianças e adolescentes apresentaram mais amadurecimento e melhores habilidades na tomada de decisão (Hutchinson \& Spangler-Hirsch, 1989), comportamento positivo e uso de estratégias de solução de problemas (Leve \& Fagot, 1997), possuíram altos índices de saúde mental e física (Hanson, 1986) e perceberam as figuras parentais de forma mais amigável do que crianças e adolescentes inseridos em famílias intactas (Asmussen \& Larson, 1991).

3) Sem mudanças e conseqüências significativas: Incluiu os abstracts nos quais não há diferenciação entre crianças e adolescentes advindos de diferentes configurações familiares quanto ao construto investigado pelos estudos analisados, ou ainda, que apontaram pontos positivos ou negativos influenciados pelas características da amostra e não pela configuração familiar. Exemplos: crianças não diferiram quanto à adesão ao tratamento para diabetes (Harris, Greco, Wysocki, Elder \& White, 1999); adolescentes não diferiram na avaliação de bem-estar psicológico (Wagner \& cols., 1999); funcionamento familiar influenciou a forma de ajustamento psicossocial de adolescentes (Hops, Tildesley, Lichtenstein, Ary \& cols., 1990); e não houve diferença entre as configurações familiares quanto à presença do transtorno depressivo em adolescentes (Patten, Gillin, Farkas \& cols., 1997).

4) Discussão das mudanças e conseqüências significativas: Diz respeito àqueles abstracts que discutiram as vivências e percepções de crianças, adolescentes e famílias, ou ainda as transformações sociais, não apresentando a direção positiva ou negativa das conseqüências das diferentes configurações familiares no desenvolvimento de crianças e adolescentes e, portanto, não apresentando resultados conclusivos. Por exemplo: o aumento nas taxas de divórcio e recasamento e as transformações sociais em decorrência dos arranjos familiares (Tasker \& Richards, 1994; Taylor, 1987); e a importância da forma como as separações e recasamentos são manejados para o desenvolvimento de crianças e adolescentes (Swick, 1984). Houve ainda abstracts que descreveram a percepção de crianças sobre o recasamento e divórcio (Frizzo, 2005) e exploraram o efeito psicológico das separações (Tusenius, 1989), entre outros, sem, no entanto, apresentar um posicionamento quanto às conseqüências das mesmas.

A Tabela 3 apresenta o cruzamento do conteúdo temático por década. Os resultados encontrados apontaram que, no total, no que se refere ao conteúdo temático dos abstracts mapeados houve um predomínio (34,94\%) de artigos que não evidenciaram mudanças e conseqüências significativas positivas ou negativas 
entre famílias nucleares e de diferentes configurações familiares para o desenvolvimento de crianças e adolescentes. Houve também uma evidência de abstracts que não indicaram tendências negativas ou positivas para o desenvolvimento de crianças e adolescentes $(34,94 \%)$, mas apontaram as vivências, percepções e os possíveis manejos, não apresentando efeitos negativos ou positivos conclusivos. A análise dos conteú- dos temáticos por década indicou que nas décadas 1980 e 1990, houve um predomínio de estudos com conteúdo de discussão das mudanças e conseqüências significativas (43\% e 33,94\%, respectivamente). $\mathrm{Na}$ década de 2000, proporcionalmente comparada à década anterior, predominaram estudos que enfocaram as mudanças e conseqüências negativas ocorridas nas diferentes configurações familiares $(41,04 \%)$.

\section{TABELA 3}

Freqüências e Percentuais do Conteúdo Temático por Década

\begin{tabular}{ccccccccccccc}
\hline Conteúdos Temáticos & \multicolumn{1}{c}{1950} & \multicolumn{1}{c}{1970} & \multicolumn{1}{c}{1980} & \multicolumn{2}{c}{1990} & \multicolumn{2}{c}{2000} & \multicolumn{3}{c}{ Total } \\
\hline & $\mathrm{f}$ & $\%$ & $\mathrm{f}$ & $\%$ & $\mathrm{f}$ & $\%$ & $\mathrm{f}$ & $\%$ & $\mathrm{f}$ & $\%$ & $\mathrm{f}$ & $\%$ \\
Mud./Conseq. Positivas & - & - & 1 & 12,5 & 9 & 8,41 & 15 & 9,09 & 12 & 8,95 & 37 & 8,91 \\
Mud./Conseq. Negativas & - & - & 4 & 50 & 28 & 26,17 & 52 & 31,51 & 55 & 41,04 & 139 & 33,5 \\
$\quad$ Sem Mud./Conseq. & - & - & - & - & 24 & 22,43 & 42 & 25,45 & 28 & 20,89 & 94 & 22,65 \\
$\quad$ Significativas & & & & & & & & & & & & \\
$\quad \begin{array}{c}\text { Discussão das } \\
\text { Mud./Conseq. }\end{array}$ & 1 & 100 & 3 & 37,5 & 46 & 42,99 & 56 & 33,94 & 39 & 29,1 & 145 & 34,94 \\
$\quad$ Significativas & & & & & & & & & & & \\
\hline$\quad$ Total & 1 & 100 & 8 & 100 & 107 & 100 & 165 & 100 & 134 & 100 & 415 & 100 \\
\hline
\end{tabular}

Entre os estudos que apontaram as mudanças e conseqüências das diferentes configurações familiares para o desenvolvimento de crianças e adolescentes, é interessante notar que sobressaíram as mudanças e conseqüências negativas em relação às positivas em todas as décadas. Estes resultados corroboram os estudos que mostraram os efeitos negativos dessas diferentes configurações familiares, que destacam o choque doloroso e angustiante vivenciado por crianças e adolescentes inseridas nessas famílias (Carter \& McGoldrick, 2001; Wallestein, 1985), os problemas de ajustamento psicológico e social (Parish \& Necessary, 1994; Walsh, 1993) e as modificações no desenvolvimento cognitivo daqueles (Ramires, 2002). As modificações na rede de convivência, de apoio e de cuidado, na quantidade e qualidade de contato e de vínculo com os filhos, a inclusão de novos parceiros, entre outros aspectos, são variáveis intimamente associadas ao ajustamento emocional. Parece ser consenso na literatura que a separação/divórcio, o recasamento e a monoparentalidade provocam uma reorganização da vida afetiva, social e econômica, tanto dos adultos quanto de crianças e adolescentes (Ramires, 2004). Entretanto, é importante lembrar que reorganização não implica somente efeitos negativos podendo representar também um potencial de crescimento às crianças e adolescentes e suas famílias.

\section{CONSIDERAÇÕES FINAIS}

O levantamento de estudos sobre famílias separadas/divorciadas, recasadas e monoparentais, e seus efeitos sobre o desenvolvimento de crianças e adolescentes permitiu identificar as principais características da produção científica nesse campo. Percebeu-se que os estudos sobre essa temática surgiram na década de 1950, havendo um acréscimo progressivo nas últimas décadas. Destaca-se um maior número de publicações na década de 1990, tanto no âmbito internacional quanto nacional, e que os anos 2000 também têm se destacado pelo número de produções científicas, quando comparado proporcionalmente às décadas anteriores. Acredita-se que o destaque observado nos anos 1990 tenha ocorrido em função de uma possível abertura e disposição, tanto dessas famílias quanto dos pesquisadores em acessar o contexto familiar em transição.

No que se refere à produção científica nacional, observou-se um crescimento no número de publicações, acompanhando a tendência internacional. Estes artigos são caracterizados, na sua maioria, como empíricos, quantitativos e de discussão sobre as mudanças e conseqüências das diferentes configurações familiares. Entretanto, a produção mostra-se ainda incipiente, tornando-se necessário desenvolver pesquisas que investiguem de maneira específica o impacto dessas configurações no desenvolvimento de crianças e 
adolescentes. Pode-se inferir que a carência de pesquisas no Brasil, verificada nesse estudo, deve-se a dois motivos. Primeiramente, tendo em vista que este mapeamento incluiu apenas periódicos indexados, é possível que a produção brasileira sobre o tema esteja inserida em periódicos não indexados ou, ainda, em capítulos de livros desenvolvidos por grupos de pesquisas em todo o país. Assim, um levantamento que inclua todos os veículos de publicação torna-se importante para o real mapeamento do campo no Brasil. O outro motivo considerado está relacionado ao tabu e aos valores predominantemente conservadores presentes na sociedade brasileira (Bucher \& Rodrigues, 1990), podendo influenciar na motivação e disposição dos pesquisadores a se inserirem em contextos familiares não considerados "modelos válidos de família", conforme apontam Narvaz e Koller (2005, p. 131).

No que tange ao método científico, percebeu-se uma evidência, tanto em nível internacional quanto nacional, de um maior número de estudos empíricos quando comparados aos de revisão de literatura sobre o tema. Quanto ao tipo de configuração familiar abordado, observou-se predomínio da monoparentalidade nos artigos internacionais, seguido pelos abstracts que falavam de pelo menos três configurações, de processo de divórcio/separação e, por fim, de recasamento.

Quanto à amostra, observou-se que há um maior número de investigações que não especificaram a faixa etária da amostra envolvida, o que não permitiu mapeá-la detalhadamente. Dos estudos que especificaram a faixa etária dos filhos, observou-se um predomínio de amostras envolvendo adolescentes em comparação com crianças. Este número de pesquisas que enfocou a adolescência pode ser entendido em função de este período do ciclo de vida ser um processo que ocorre durante o desenvolvimento do indivíduo (Levisky, 1998), caracterizando-se como de transição e de adaptações às regras sociais. Por essa razão, é possível compreender que os efeitos das diferentes configurações para adolescentes podem ser mais expressivos e desencadear sintomas psicopatológicos, maximizando os conflitos e as reorganizações nesse momento, e reforçando o interesse científico por essa população. Por outro lado, também se pode pensar que o fato de os adolescentes apresentarem um desenvolvimento cognitivo mais maduro e também a conveniência por participar de atividades novas e auto-respondíveis podem ser fatores que facilitem o desenvolvimento de pesquisas junto aos mesmos.

Cabe destacar, enquanto limitação do presente estudo, que a análise dos dados está baseada apenas em abstracts de periódicos científicos indexados, podendo não dar a idéia da totalidade da produção científica. Através do mapeamento e da avaliação de abstracts de periódicos científicos, evidenciou-se também uma pluralidade de estudos sobre o tema. Esta sistematização permitiu identificar o crescente avanço do campo científico sobre o conteúdo temático e as transformações de paradigmas que a organização familiar foi sofrendo ao longo dos tempos. Além disso, possibilitou inferir que não há consenso na literatura quanto aos efeitos das diferentes configurações para o desenvolvimento de crianças e adolescentes. Podem-se encontrar artigos que apontaram os prejuízos e outros que mostraram os aspectos positivos, mas a maioria apresentou uma posição neutra, descrevendo, problematizando e refletindo acerca dos fatores que podem influenciar os efeitos dessas configurações sobre o desenvolvimento.

Em contrapartida, ainda chama a atenção a tendência predominante de artigos que enfocaram as mudanças e conseqüências negativas em relação às positivas das diferentes configurações investigadas nesse artigo. É possível pensar que, de fato, as transições vivenciadas pelas famílias separadas/divorciadas, recasadas e monoparentais estão carregadas de sofrimento e inúmeros prejuízos ao desenvolvimento (Carter \& McGoldrick, 2001; Hetherington \& Stanley-Hagan, 1999; Mazur, 1993; Parish \& Necessary, 1994; Ramires, 2002; 2004; Wallestein, 1985; Walsh, 1993; Wagner, 2002; Wagner \& cols., 1999; Wagner \& cols., 1997). Por outro lado, essas pesquisas ainda podem estar sob influência da força social que estigmatiza as famílias que vivem nessas configurações, não considerando o potencial de saúde presente nas mesmas.

A literatura contemporânea tem apontado o potencial de saúde que o sistema familiar tem para encontrar alternativa na solução dos seus problemas e conseguir conter os efeitos destrutivos em seus membros (Féres-Carneiro, 1992). A plasticidade das relações no núcleo familiar pode gerar uma infinidade de recursos promotores de saúde. Uma família facilitadora do crescimento emocional e promotora de saúde não é aquela com ausência de conflitos (Wagner \& cols., 1999). Bons níveis de saúde familiar, muitas vezes, encontram-se associados a núcleos que favorecem tanto a expressão da agressividade, de raiva e hostilidade quanto de carinho, ternura e afeto. Para Walsh (1996), a capacidade de responder positivamente aos desafios enfrentados pelas famílias está relacionada ao desenvolvimento de características de resiliência dos membros e da própria família. 
A separação/divórcio, monoparentalidade e recasamento são períodos de transição no ciclo de vida da família que acarretam implicações diretas para os membros familiares envolvidos. Sendo assim, esses períodos não devem ser entendidos como algo que caracterize o fim ou a dissolução da instituição da família, mas como um período de reestruturação de papéis e de sistemas de valores, exigindo de seus membros uma adaptação às mudanças no relacionamento e na estrutura familiar (Costa, 1991; Costa \& Féres-Carneiro, 1992; Grzybowski, 2002; Yunes, 2001). Os diversos campos do conhecimento precisam refletir sobre essas diferentes configurações familiares associadas à herança dos modelos tradicionais, no sentido de procurar a qualidade de vida dos membros numa organização diferente, minimizando os efeitos negativos sobre o desenvolvimento de crianças e adolescentes. Repensar os novos valores sociais e culturais, preconizando a promoção de saúde entre os membros da família, especialmente para crianças e adolescentes, mais do que a manutenção das configurações familiares, é tarefa básica dos diferentes profissionais que atentam para a saúde no ambiente familiar.

\section{REFERÊNCIAS}

Obs.: Referências precedidas de um asterisco indicam estudos incluídos na revisão.

*Amato, P. P. (1995). Children's adjustment to divorce: Theories, hypotheses, and empirical support. Journal of Marriage and Family, 55, 628-640.

*Asmussen, L., \& Larson, R. (1991). The quality of family time among young adolescents in single-parent and married-parent families. Journal of Marriage and the Family, 53, 1021-1030.

*Barocas, R., Seifer, R., \& Sameroff, A. (1985). Defining environmental risk: Multiple dimensions of psychological vulnerability. American Journal of Community Psychology, 13, 433447.

Benz, J. N., \& Newman, J. (1998). Qualitative-quantitative research: A false dichotomy. Em Research methodology: Qualitative-quantitative (pp. 1-13). Carbondale, IL: Southern Illinois University Press.

*Bucher, J. S. N., \& Rodrigues, M. A. (1990). Recasamento e recomposição familiar: Questões metodológicas, de linguagem e das teorias. Psicologia: Teoria e Pesquisa, 6, 155-169.

Carter, B., \& McGoldrick, M. (2001). As mudanças no ciclo de vida da família: Uma estrutura para a terapia familiar $\left(2^{\mathrm{a}} \mathrm{ed}\right.$.) (Veronese, M. A., Trad.). Porto Alegre: Artes Médicas. (Originalmente publicado em 1989)

*Costa, L. (1991). A família descasada: Uma nova perspectiva. Psicologia: Teoria e Pesquisa, 7, 229-246.

*Costa, L., \& Féres-Carneiro, T. (1992). Reorganizações familiares: As possibilidades de saúde a partir da separação conjugal. Psicologia: Teoria e Pesquisa, 8, 495-504.
Creswell, J. (1994). Research design: Qualitative \& quantitative approaches. London: SAGE Publications.

De Antoni, C. (2005). Coesão e hierarquia em famílias com história de abuso físico. Tese de doutorado não-publicada, Curso de Pós-Graduação em Psicologia de Desenvolvimento, Universidade Federal do Rio Grande do Sul. Porto Alegre, RS.

Dessen, M. A., \& Braz, M. P. (2005). A família e suas interrelações com o desenvolvimento humano. Em M. A. Dessen \& A. L. Costa Jr. (Orgs), A ciência do desenvolvimento humano: Tendências atuais e perspectivas futuras (pp. 113-131). Porto Alegre: Artmed.

*Erel, O., \& Burman, B. (1995). Interrelatedness of marital relations and parent-child relations: A meta-analytic review. Psychological Bulletin, 118, 108-132.

Fachin, R. (2001). Em busca da família do novo milênio: Uma reflexão crítica sobre as origens históricas e as perspectivas do Direito de Família brasileiro contemporâneo. Rio de Janeiro: Renovar.

*Féres-Carneiro, T. (1992). Família e saúde mental. Psicologia: Teoria e Pesquisa, 8, 485-493.

*Féres-Carneiro, T. (1998). Casamento contemporâneo: O difícil convívio da individualidade com a conjugalidade. Psicologia: Reflexão e Crítica, 11, 379-394.

*Féres-Carneiro, T. (2003). Separação: O doloroso processo de dissolução da conjugalidade. Estudos de Psicologia, 8, 367374.

Ferreira, N. S. (2002). As pesquisas denominadas "Estado da Arte". Educação \& Sociedade, 23, 257-272.

Figueira, S. A. (1986). O "moderno" e o "arcaico" na nova família brasileira: Notas sobre a dimensão invisível da mudança social. Em S. A. Figueira (Org), Uma nova família? O moderno e o arcaico na família de classe média brasileira. Rio de Janeiro: Zahar.

*Fletcher, D. R. (2004). Employing drug users: Individual and systemic barriers to rehabilitation. Community, Work and Family, 7, 117-119.

*Frizzo, G. (2005). As novas configurações familiares e a depressão materna: Análise do filme "Um Grande Garoto". Pensando Famílias, 7, 135-146.

*Fonseca, C. W. (2002). Mãe é uma só? Reflexões em torno de casos brasileiros. Psicologia USP, 13, 49-68.

Godoy, A. S. (1995a). Introdução à pesquisa qualitativa e suas possibilidades. Revista de Administração de Empresas, 35(2), 57-63.

Godoy, A. S. (1995b). Pesquisa qualitativa: Tipos fundamentais. Revista de Administração de Empresas, 35, (3), 20-29.

*Goldani, A. M. (1994). As famílias brasileiras: Mudanças e perspectivas. Cadernos de Psicologia, 91, 7-22.

*Grossman, M., \& Rowat, K. (1995). Parental relationships, coping strategies, received support and well-being in adolescents of separated or divorced and married parents. Research in Nursing \& Health, 18, 249-261.

*Grzybowski, L. (2002). Famílias monoparentais: Mulheres divorciadas chefes de famílias. Em A. Wagner (Org.), Família em cena: Tramas, dramas e transformações (pp. 39-53). Petrópolis: Ed. Vozes. 
*Guidubaldi, J., \& Perry, J. (1984). Divorce, socioeconomic status, and children's cognitive-social competence at school entry. American Journal of Orthopsychiatry, 54, 459-468.

*Hanson, S. (1986). Healthy single parent families. Family Relations: Journal of Applied Family and Child Studies, 35, 125132.

*Harris, M., Greco, P., Wysocki, T., Elder, D., \& White, N. (1999). Adolescents with diabetes from single-parent, blended, and intact families: Health-related and family functioning. Families, Systems and Health, 17, 181-196.

*Hetherington, E. M., \& Stanley-Hagan, M. (1999). The adjustment of children with divorced parents: A risk and a resiliency perspective. The Journal of Child Psychology and Psychiatry and Allied Disciplines, 40, 129-140.

*Hops, H., Tildesley, E., Lichtenstein, E., Ary, D., \& cols. (1990). Parent-adolescent problem-solving interactions and drug use. American Journal of Drug and Alcohol Abuse, 16, 239-258.

*Hutchinson, R., \& Spangler-Hirsch, S. (1989). Children of divorce and single-parent lifestyles: Facilitating well-being. Journal of Divorce, 12, 5-24.

IBGE (2002/2003). Censo Nacional Instituto Brasileiro de Geografia e Estatística. Retirado em 30 de abril de 2007 do Instituto Brasileiro de Geografia e Estatística, em http://www. ibge.gov.br/home/presidencia/noticias/17122003registrocivilht ml.shtm

Kaslow, F. W., \& Schwartz, L. L. (1995). As dinâmicas do divórcio. (M. Lopes, \& M. Carbajal, Trad.). São Paulo: Editorial Psy. (Originalmente publicado em 1987)

Kreppner, K. (2000). The child and the family: Interdependence in developmental pathways. Psicologia: Teoria e Pesquisa, 16, 11-22.

*Kroes, M., Kalff, A., Steyaert, J., Kessels, A., Feron, F., Hendriksen, J., Van-Zeben, T., Troost, J., Jolles, J., \& Vles, J. (2002). A longitudinal community study: Do psychosocial risk factors and Child Behavior Checklist scores at 5 years of age predict psychiatric diagnoses at a later age? Journal of the American Academy of Child and Adolescent Psychiatry, 41, 955-963.

*Lalloo, R., Sheiham, A., \& Nazroo, J. (2003). Behavioural characteristics and accidents: Findings from the Health Survey for England, 1997. Accident Analysis and Prevention, 35, 661-667.

*Leve, L., \& Fagot, B. (1997). Gender-role socialization and discipline processes in one- and two-parent families. Sex Roles, $36,1-21$.

Levisky, D. (1998). Adolescência: Reflexões psicanalíticas. São Paulo: Casa do Psicólogo.

* Mazur, E. (1993). Developmental differences in children's understanding of marriage, divorce and remarriage. Journal of Aplied Developmental Psychology, 14, 191-212.

Minuchin, S. (1982). Famílias: Funcionamento e tratamento. Porto Alegre: Artes Médicas.

Narvaz, M., \& Koller, S. H. (2005). A invenção da família. Pensando Famílias, 7, 121-134.

Osório, L. C. (1992). Adolescente hoje. Porto Alegre: Artes Médicas.

Osório, L. C. (2002). Casais e famílias: Uma visão contemporânea. Porto Alegre: Artes Médicas.
*Parish, T., \& Necessary, J. (1994). Do attitudinal and behavioral ratings of family members vary across familial configurations? Journal Adolescence, 29, 649-652.

*Patten, C., Gillin, C., Farkas, A., Gilpin, E., Berry, C., \& Pierce, J. (1997). Depressive symptoms in California adolescents: Family structure and parental support. Journal of Adolescent Health, 20, 271-278.

Perrot, M. (1993). O nó e o ninho. São Paulo: Abril.

*Pollinger, J., Samuels, L., \& Stadolnik, R. (2005). A comparative study of the behavioral, personality, and fire history characteristics of residential and outpatient adolescents (ages 12-17) with firesetting behaviors. Adolescence, 40, 345-353.

*Ramires, V. R. (2004). As transições familiares: A perspectiva de crianças e pré-adolescentes. Psicologia em Estudo, 9, 183-193.

Ramires, V. R. R. (2002). O amor e suas vicissitudes: As concepções de crianças e adolescentes. Tese de Doutorado nãopublicada, Programa de Pós-Graduação em Psicologia Clínica, Pontifícia Universidade Católica de São Paulo. São Paulo, SP.

*Rizzini, I. (2002). Crianças, adolescentes e famílias: Tendências e preocupações globais. Interação em Psicologia, 6, 45-47.

Swick, K. (1984). Understanding special needs families. Journal of Instructional Psychology, 11, 37-47.

Tasker, F. L., \& Richards, M. P. M. (1994). Adolescents' attitudes toward marriage and marital prospects after parental divorce: A review. Journal of Adolescent Research, 9, 340-362.

Taylor, R. H. (1987). Black youth in crisis. Journal of Social Relations, 14, 106-133.

Tusenius, A. A. (1989). The effects of divorce on children. Tijdschrift voor Psychiatrie, 31, 15-28.

Wagner, A. (2002). Família em cena: Tramas, dramas e transformações. Petrópolis: Ed. Vozes.

*Wagner, A, Falcke, D., \& Meza, E. (1997). Crenças e valores dos adolescentes acerca de família, casamento, separação e projetos de vida. Psicologia: Reflexão e Crítica, 10, 155-167.

*Wagner, A; Ribeiro, L., Arteche, A., \& Bornholdt, E. (1999). Configuração familiar e o bem-estar psicológico dos adolescentes. Psicologia: Reflexão e Crítica, 12, 147-156.

Wallerstein, J. (1985). Children of divorce: Emerging trends. Psychiatric Clinics of North America, 4, 837-855.

Wallerstein, J. S., \& Kelly, J. B. (1998). Sobrevivendo à separação. (M. A. V. Veronese, Trad.). Porto Alegre: ArtMed. (Originalmente publicado em 1996)

Walsh, F. (1993). Normal family processes. New York: Guilford Press.

*Walsh, F. (1996). The concept of family resilience: Crisis and challenge. Family Process, 35, 261-281.

*Weissman, A., Jogerst, G., \& Dawson, J. (2003). Community characteristics associated with child abuse in Iowa. Child Abuse and Neglect, 27, 1145-1159.

Winnicott, D. (2001). A família e o desenvolvimento individual ( $2^{\mathrm{a}}$ ed.) (M. B. Cipolla, Trad.). São Paulo: Martins Fontes. (Originalmente publicado em 1965)

Yunes, M. A. M. (2001). A questão triplamente controvertida da resiliencia em familias de baixa renda. Tese de doutorado não publicada, Programa de Pós-Graduação em Psicologia da Educação, Pontifícia Universidade Católica de São Paulo. São Paulo, SP. 
Recebido: $25 / 09 / 2007$

Última revisão: $30 / 01 / 2008$

Aceite final: $20 / 02 / 2008$

\section{Sobre as autoras:}

Débora de Oliveira: Psicóloga, Mestre e Doutoranda em Psicologia da Universidade Federal do Rio Grande do Sul (UFRGS).

Aline Cardoso Siqueira: Psicóloga, Mestre e Doutoranda em Psicologia da Universidade Federal do Rio Grande do Sul (UFRGS).

Débora Dalbosco Dell'Aglio: Doutora em Psicologia do Desenvolvimento, pesquisadora do CNPq e Docente no PPG em Psicologia da Universidade Federal do Rio Grande do Sul (UFRGS).

Rita de Cássia Sobreira Lopes: Doutora pela Universidade de Londres (Inglaterra), pesquisadora do CNPq e Docente do PPG Psicologia da Universidade Federal do Rio Grande do Sul (UFRGS).

Endereço para correspondência: Rita de Cássia Sobreira Lopes: Instituto de Psicologia, Universidade Federal do Rio Grande do Sul Rua Ramiro Barcelos, 2600, Sala 108. 90035-003 - Porto Alegre, RS. Endereço eletrônico: sobreiralopes@portoweb.com.br. 